\title{
Standard completeness for extensions of MTL: an automated approach
}

\author{
Paolo Baldi Agata Ciabattoni Lara Spendier \\ Technische Universität Wien, Austria
}

\begin{abstract}
We provide general conditions on hypersequent calculi that guarantee standard completeness for the formalized logics. These conditions are implemented in the PROLOG system AxiomCalc that takes as input any suitable axiomatic extension of Monoidal T-norm Logic MTL and outputs a hypersequent calculus for the logic and the result of the check. Our approach subsumes many existing results and allows for the computerized discovery of new fuzzy logics.
\end{abstract}

\section{Introduction}

Standard completeness, that is completeness of a logic with respect to algebras based on truth values in $[0,1]$, has received increasing attention in the last few years. In a standard complete logic connectives are interpreted by suitable functions on $[0,1]$, and this makes it a fuzzy logic in the sense of [11]. For example conjunction and implication can be interpreted by a t-norm ${ }^{1}$ and its residuum or by classes of t-norms. Gödel and Łukasiewicz being prominent examples of logics based on particular t-norms, while e.g. Monoidal t-norm logic MTL [8] is based on the class of left-continuous t-norms. MTL is a useful basic system as many interesting logics, including Gödel and Łukasiewicz logics, can be obtained by extending MTL with suitable properties expressed as Hilbert axioms.

Checking or discovering whether a logic is standard complete is sometimes a challenging task which deserves a paper for each specific logic, e.g., $[5,10,12]$. It is traditionally established by semantic techniques which are inherently logicspecific. Given a logic $\mathbf{L}$ described in a Hilbert-style system, semantic proofs usually consist of the following four steps (see, e.g., $[5,7,8,10,11,15])$ :

1. The algebraic semantics of the logic is identified ( $\mathcal{L}$-algebras).

2. It is shown that if a formula is not valid in an $\mathcal{L}$-algebra, then it is not valid in a countable $\mathcal{L}$-chain (linearly ordered $\mathcal{L}$-algebra).

3. It is shown that any countable $\mathcal{L}$-chain can be embedded into a countable dense $\mathcal{L}$-chain by adding countably many new elements to the algebra and extending the operations appropriately. This establishes rational completeness: a formula is derivable in $\mathbf{L}$ iff it is valid in all dense $\mathcal{L}$-chains.

4. Finally, a countable dense $\mathcal{L}$-chain is embedded into a standard $\mathcal{L}$-algebra, that is an $\mathcal{L}$-algebra with lattice reduct $[0,1]$, using a Dedekind-MacNeillestyle completion.

\footnotetext{
${ }^{1}$ T-norms are the main tool in fuzzy set theory to combine vague information.
} 
The crucial step 3. above (rational completeness) is the most difficult to establish as it relies on finding the right embedding, if any. A different approach to step 3. was proposed in [13] by using proof-theoretic techniques. The idea in [13] is that the admissibility of a particular syntactic rule (called density) in a logic $\mathbf{L}$ can lead to a proof of rational completeness for $\mathbf{L}$. This is for instance the case when $\mathbf{L}$ is any axiomatic extension of MTL. Introduced by Takeuti and Titani in [17] the density rule formalized Hilbert-style has the following form

$$
\frac{(A \rightarrow p) \vee(p \rightarrow B) \vee C}{(A \rightarrow B) \vee C}
$$

where $p$ is a propositional variable not occurring in $A, B$, or $C$. Ignoring $C$, this can be read contrapositively as saying (very roughly) "if $A>B$, then $A>p$ and $p>B$ for some $p$ "; hence the name "density" and the intuitive connection with rational completeness.

The new approach was used in [13] to establish standard completeness for various logics for which semantic techniques do not appear to work. Following this method, to establish standard completeness for a logic $\mathbf{L}$ we need to

(a) define a suitable proof system $H L$ for $\mathbf{L}$ extended with the density rule

(b) check that this rule is eliminable (or admissible) in $H L$, i.e. that density does not enlarge the set of provable formulas

(c) standard completeness may then be obtained in many cases (but not in general) by means of the Dedekind-MacNeille completion.

A convenient proof system HMTL for MTL uses hypersequents, which are a simple generalization of Gentzen sequents, see [1,14].

Step (b) above (density-elimination) was shown in $[2,13]$ for various calculi, including $H M T L$. The proofs in [2,13] are calculi-specific and use heavy combinatorial arguments, in close analogy with Gentzen style cut-elimination proofs. A different method to eliminate applications of the density rule from derivations was introduced in [6]. It shows that each hypersequent calculus obtained by extending HMTL by certain sequent rules admits density-elimination. Though more general than the proofs in $[2,13]$, this result does not apply to many interesting extensions of MTL whose additional axioms require hypersequent rules; e.g., it does not apply to weak nilpotent minimum logic WNM $[4,8]$.

The aim of this paper is to automate standard completeness proofs for large classes of axiomatic extensions of MTL.

We introduce the program Axiom Calc that automates steps (a)-(c) above for propositional logics extending MTL by any Hilbert axiom within the class $\mathcal{P}_{3}$ in the syntactic classification of [4]. The main theoretical contribution of this paper is the identification of sufficient conditions on hypersequent rules that ensure standard completeness for the formalized logics. As shown in Section 3, our conditions allow indeed density-elimination (and hence, by [13], they lead to rational completeness). Standard completeness follows, being the axioms we deal with preserved under suitable forms of Dedekind-MacNeille completions [3].

Axiom Calc implements the systematic procedure in [4] to define cut-free hypersequent calculi from Hilbert systems for large classes of logics (step (a)), 
and the check of our sufficient conditions which account for steps (b) and (c) at once.

Our approach subsumes many existing results on standard completeness for specific logics and allows for the computerized discovery of new fuzzy logics. For instance, it applies to WNM and to the logics obtained by extending MTL with the axioms $\neg(\alpha \cdot \beta)^{n} \vee\left((\alpha \wedge \beta)^{n-1} \rightarrow(\alpha \cdot \beta)^{n}\right)$ for each $n \geq 2$. The latter family of logics is new and was discovered by playing with the PROLOG system AxiomCalc, available at http://www.logic.at/people/lara/axiomcalc.html.

\section{Automated proof theory for extensions of MTL}

In this section we present the system Axiom Calc and describe the calculi that it generates. The program is a PROLOG-implementation of the algorithm in [4].

The basic system we will deal with is Monoidal T-norm Logic MTL [8] which was shown in [10] to be the logic of left continuous t-norms ${ }^{2}$ and their residua. MTL is obtained by adding the prelinearity axiom $(\alpha \rightarrow \beta) \vee(\beta \rightarrow \alpha)$ to intuitionistic logic without contraction (also known as HBCK [16] or Full Lambek calculus with exchange and weakening FLew).

Formulas of MTL are built from propositional variables and the constants 0 and 1 by using $\rightarrow$ (implication), $\wedge$ (additive conjunction), · (multiplicative conjunction), and $\vee$ (disjunction). We use $\neg \alpha$ as an abbreviation for $\alpha \rightarrow 0$.

In the following $\alpha, \beta, \ldots$ will stand for both formulas and for metavariables for formulas. To distinguish between rule applications and rule schemas we will denote finite (possibly empty) multisets of formulas with $\Gamma, \Delta, \Sigma, \Theta, \Lambda$ and metavariables for multisets of formulas with $\bar{\Gamma}, \bar{\Delta}, \bar{\Sigma}, \bar{\Theta}, \bar{\Lambda}$. Metavariables $\bar{\Pi}, \bar{\Psi}$ will stand for stoups, i.e., either a formula or the empty set.

Definition 1. A hypersequent is a finite multiset $S_{1}|\ldots| S_{n}$ where each $S_{i}, i=$ $1 \ldots n$ is a sequent, called a component of the hypersequent.

The symbol "|" is intended to denote disjunction at the meta-level. In this paper we will only consider hypersequents whose components contain at most one formula on their right-hand side.

Henceforth we will denote hypersequents by $G, H$ and sequents (possibly built from metavariables) by $S_{i}, C_{i}$. The hypersequent calculus contains initial axioms, cut, and logical rules. These are as in sequent calculus, the only difference being the presence of a (possibly empty) side hypersequent $G$. Structural rules are divided into two groups: internal and external rules. The latter, which permit interaction between components, increase the expressive power of the hypersequent calculus with respect to sequent calculus.

The (cut-free) hypersequent calculus HMTL for MTL is obtained by adding Avron's communication rule (com) to the hypersequent version of the sequent

\footnotetext{
${ }^{2}$ A $t$-norm is a commutative, associative, increasing function $*:[0,1]^{2} \rightarrow[0,1]$ with identity element $1 . *$ is left continuous iff whenever $\left\{x_{n}\right\},\left\{y_{n}\right\}(n \in N)$ are increasing sequences in $[0,1]$ s.t. their suprema are $x$ and $y$, then $\sup \left\{x_{n} * y_{n}: n \in N\right\}=x * y$. The residuum of $*$ is a function $\rightarrow^{*}$ where $x \rightarrow^{*} y=\max \{z \mid x * z \leq y\}$.
} 


$$
\begin{aligned}
& \frac{G|\bar{\Gamma} \Rightarrow \alpha \quad G| \alpha, \bar{\Delta} \Rightarrow \bar{\Pi}}{G \mid \bar{\Gamma}, \bar{\Delta} \Rightarrow \bar{\Pi}}(C u t) \quad \overline{G \mid \alpha \Rightarrow \alpha}(\text { init }) \quad \overline{G \mid 0 \Rightarrow}(0 l) \\
& \frac{G|\bar{\Gamma} \Rightarrow \alpha \quad G| \bar{\Delta} \Rightarrow \beta}{G \mid \bar{\Gamma}, \bar{\Delta} \Rightarrow \alpha \cdot \beta}(\cdot r) \quad \frac{G \mid \alpha, \beta, \bar{\Gamma} \Rightarrow \bar{\Pi}}{G \mid \alpha \cdot \beta, \bar{\Gamma} \Rightarrow \bar{\Pi}}(\cdot l) \quad \frac{G \mid \bar{\Gamma} \Rightarrow \bar{\Pi}}{G \mid 1, \bar{\Gamma} \Rightarrow \bar{\Pi}}(1 l) \\
& \frac{G|\bar{\Gamma} \Rightarrow \alpha \quad G| \beta, \bar{\Delta} \Rightarrow \bar{\Pi}}{G \mid \bar{\Gamma}, \alpha \rightarrow \beta, \bar{\Delta} \Rightarrow \bar{\Pi}}(\rightarrow l) \quad \frac{G \mid \alpha, \bar{\Gamma} \Rightarrow \beta}{G \mid \bar{\Gamma} \Rightarrow \alpha \rightarrow \beta}(\rightarrow r) \quad \frac{G \mid \bar{\Gamma} \Rightarrow}{G \mid \bar{\Gamma} \Rightarrow 0}(0 r) \\
& \frac{G|\bar{\Gamma} \Rightarrow \alpha \quad G| \bar{\Gamma} \Rightarrow \beta}{G \mid \bar{\Gamma} \Rightarrow \alpha \wedge \beta}(\wedge r) \quad \frac{G \mid \alpha_{i}, \bar{\Gamma} \Rightarrow \bar{\Pi}}{G \mid \alpha_{1} \wedge \alpha_{2}, \bar{\Gamma} \Rightarrow \bar{\Pi}}(\wedge l) \quad \overline{G \mid \Rightarrow 1}(1 r) \\
& \frac{G|\alpha, \bar{\Gamma} \Rightarrow \bar{\Pi} \quad G| \beta, \bar{\Gamma} \Rightarrow \bar{\Pi}}{G \mid \alpha \vee \beta, \bar{\Gamma} \Rightarrow \bar{\Pi}}(\vee l) \frac{G \mid \bar{\Gamma} \Rightarrow \alpha_{i}}{G \mid \bar{\Gamma} \Rightarrow \alpha_{1} \vee \alpha_{2}}(\vee r) \frac{G \mid \bar{\Gamma} \Rightarrow \bar{\Pi}}{G \mid \bar{\Gamma}, \alpha \Rightarrow \bar{\Pi}}(w l) \\
& \frac{G|\bar{\Gamma} \Rightarrow \bar{\Pi}| \bar{\Gamma} \Rightarrow \bar{\Pi}}{G \mid \bar{\Gamma} \Rightarrow \bar{\Pi}}(E C) \quad \frac{G}{G \mid \bar{\Gamma} \Rightarrow \bar{\Pi}}(E W) \quad \frac{G \mid \bar{\Gamma} \Rightarrow}{G \mid \bar{\Gamma} \Rightarrow \bar{\Pi}}(w r) \\
& \frac{G\left|\bar{\Gamma}_{1}, \bar{\Delta}_{1} \Rightarrow \bar{\Pi}_{1} \quad G\right| \bar{\Gamma}_{2}, \bar{\Delta}_{2} \Rightarrow \bar{\Pi}_{2}}{G\left|\bar{\Gamma}_{1}, \bar{\Gamma}_{2} \Rightarrow \bar{\Pi}_{1}\right| \bar{\Delta}_{1}, \bar{\Delta}_{2} \Rightarrow \bar{\Pi}_{2}}(\mathrm{com})
\end{aligned}
$$

Table 1. Hypersequent calculus $H M T L$ for MTL

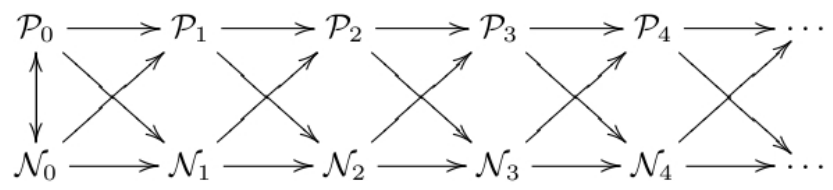

Fig. 1. Classification $\left(\mathcal{N}_{n}, \mathcal{P}_{n}\right)[4]$

calculus for FLew, see Table 1. (Note that a sequent rule can be easily transformed into a hypersequent rule by adding the context $G$ everywhere).

A classification of Hilbert axioms in the language of FLew was introduced in [4]. It is based on classes $\left(\mathcal{N}_{n}, \mathcal{P}_{n}\right)$ which intuitively account for the difficulty to deal with the axioms proof theoretically.

The general grammar for determining the class of each axiom is as follows ( $\mathcal{A}$ is the set of atomic formulas):

$$
\begin{aligned}
& \mathcal{P}_{0}::=\mathcal{N}_{0}::=\mathcal{A} \\
& \mathcal{P}_{n+1}::=\mathcal{N}_{n}\left|\mathcal{P}_{n+1} \cdot \mathcal{P}_{n+1}\right| \mathcal{P}_{n+1} \vee \mathcal{P}_{n+1} \mid 1 \\
& \mathcal{N}_{n+1}::=\mathcal{P}_{n}\left|\mathcal{P}_{n+1} \rightarrow \mathcal{N}_{n+1}\right| \mathcal{N}_{n+1} \wedge \mathcal{N}_{n+1} \mid 0
\end{aligned}
$$

A graphical representation of this classification is depicted in Figure 1. Note that the arrows $\rightarrow$ stand for inclusions $\subseteq$ of the classes. 


\begin{tabular}{|c|c|c|c|}
\hline Class & Axiom & Rule (cf. Table 3) & Logic \\
\hline $\mathcal{N}_{2}$ & $\alpha \rightarrow \alpha \cdot \alpha$ & $(c)$ & Gödel logic G \\
\hline $\mathcal{P}_{2}$ & $\alpha \vee \neg \alpha$ & $(\mathrm{em})$ & Classical Logic CL \\
\hline $\mathcal{P}_{3}$ & $\neg \alpha \vee \neg \neg \alpha$ & $($ lq) & SMTL \\
& $\alpha \vee(\alpha \rightarrow \beta) \vee(\alpha \wedge \beta \rightarrow \gamma)$ & $($ bc2) & 3-valued G (with $(c))$ \\
& $\neg(\alpha \cdot \beta) \vee(\alpha \wedge \beta \rightarrow \alpha \cdot \beta)$ & $($ wnm $)$ & WNM \\
& $\neg(\alpha \cdot \beta)^{n} \vee\left((\alpha \wedge \beta)^{n-1} \rightarrow(\alpha \cdot \beta)^{n}\right)$ & $(\text { wnm })^{n}$ & new! \\
\hline
\end{tabular}

Table 2. Some axioms and their corresponding logics

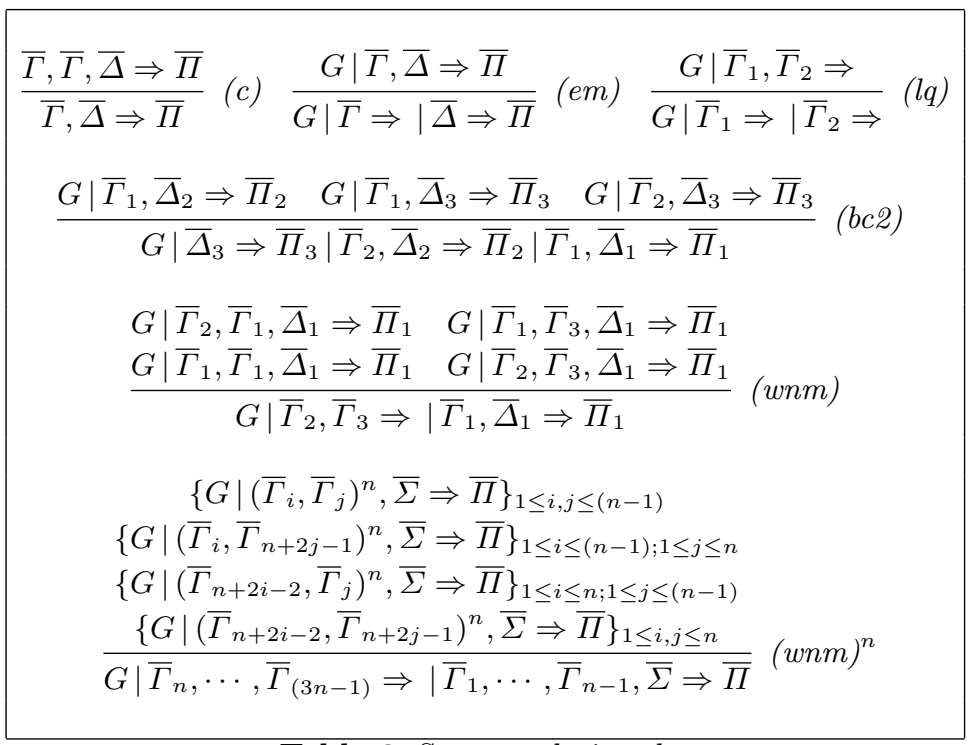

Table 3. Some analytic rules

Paper [4] also contains a procedure to transform axioms within the classes ${ }^{3}$ $\mathcal{N}_{2}, \mathcal{P}_{2}$ and $\mathcal{P}_{3}$ into equivalent rules which preserve cut-admissibility once added to the (hypersequent version of) the calculus for FLew.

Example 1. The axiom $\neg(\alpha \cdot \beta)^{2} \vee\left((\alpha \wedge \beta) \rightarrow(\alpha \cdot \beta)^{2}\right)$ (that is $\neg(\alpha \cdot \beta \cdot \alpha \cdot \beta) \vee$ $((\alpha \wedge \beta) \rightarrow(\alpha \cdot \beta \cdot \alpha \cdot \beta)))$ is in the class $\mathcal{P}_{3}$. The equivalent rule generated by the algorithm in [4] is

$$
\frac{\left\{G \mid \bar{\Gamma}_{1}^{2}, \bar{\Gamma}_{i}^{2}, \bar{\Sigma} \Rightarrow \bar{\Pi}\right\}_{1 \leq i \leq 5} \quad\left\{G \mid \bar{\Gamma}_{i}^{2}, \bar{\Gamma}_{i+1}^{2}, \bar{\Sigma} \Rightarrow \bar{\Pi}\right\}_{2 \leq i \leq 4} \quad G \mid \bar{\Gamma}_{2}^{2}, \bar{\Gamma}_{5}^{2}, \bar{\Sigma} \Rightarrow \bar{\Pi}}{G\left|\bar{\Gamma}_{2}, \bar{\Gamma}_{3}, \bar{\Gamma}_{4}, \bar{\Gamma}_{5} \Rightarrow\right| \bar{\Gamma}_{1}, \bar{\Sigma} \Rightarrow \bar{\Pi}}(\text { wnm })^{2}
$$

where the notation $X^{k}$ within inference rules stands for $X, \ldots, X k$ times, with $k \geq 0$. For further examples see Tables 2 and 3 .

\footnotetext{
${ }^{3}$ An explicit description of the axioms in these classes is given in Appendix.
} 


\subsection{Density and convergent rules}

The density rule was introduced by Takeuti and Titani in their axiomatization of first-order Gödel logic [17]. In hypersequent calculi (an instance of) this rule has the form:

$$
\frac{G^{\prime}|\Sigma, p \Rightarrow \Pi| \Lambda \Rightarrow p}{G^{\prime} \mid \Sigma, \Lambda \Rightarrow \Pi}(D)
$$

where $p$ is a propositional variable not occurring in $\Sigma, \Lambda, \Pi$ or $G^{\prime}$ ( $p$ is an eigenvariable). Note that adding the density rule to a hypersequent calculus can have a dramatic effect. Consider, e.g., HMTL + (em), i.e., HMTL extended with the rule (em) (cf. Table 3 ): by adding $(D)$ we are able to prove the empty sequent as follows:

$$
\frac{\frac{\overline{p \Rightarrow p}}{p \text { (init) }}}{\frac{p \mid \Rightarrow p}{\Rightarrow}}(\text { em })
$$

A similar situation arises for $H M T L+(b c 2)+(c)$. This is no surprise since, as shown in [13], the addition and subsequent elimination of $(D)$ from an extension of HMTL leads to rational completeness for the formalized logic, and the two calculi above formalize logics that are not rational complete: classical and 3valued Gödel logic (see Table 2).

However, for many extensions of $H M T L$, adding $(D)$ has no effect on which hypersequents are derivable: applications of $(D)$ can be eliminated from derivations. Below we identify properties that, when satisfied by hypersequent rules generated using the algorithm in [4], ensure density-elimination of the corresponding extensions of $H M T L$. Rules satisfying these properties will be called convergent.

Given a sequent $S$ henceforth we will denote by $L(S)$ its left hand side and by $R(S)$ its right hand side. Let $S:=\Gamma_{1}, \Gamma_{2} \Rightarrow \Pi$, we indicate by $S\left[{ }^{\Gamma_{1}} / \Lambda\right]^{l}[\Pi / \Sigma \Rightarrow \Psi]^{r}$ the sequent $\Lambda, \Gamma_{2}, \Sigma \Rightarrow \Psi$. The notations apply also to metasequents, i.e., sequents built from metavariables.

In what follows we will refer to any hypersequent rule generated by the procedure in [4] as completed.

Definition 2. Let $(r)$ be a completed hypersequent rule:

$$
\frac{G\left|S_{1} \quad \ldots \quad G\right| S_{m}}{G\left|C_{1}\right| \ldots \mid C_{q}}
$$

Let $G \mid S_{i}$ and $G \mid S_{j}$ be among its premises.

(0-pivot) $G \mid S_{i}$ is a 0-pivot if there is an $s \in\{1, \ldots, q\}$ such that $R\left(S_{i}\right)=R\left(C_{s}\right)$ and the different metavariables in $L\left(S_{i}\right)$ are contained in those of $L\left(C_{s}\right)$.

(n-pivot) $G \mid S_{j}$ is an n-pivot for $G \mid S_{i}$, for $n>0$, if the following conditions hold:

- $G \mid S_{j}$ is a 0-pivot

- $R\left(S_{i}\right)=R\left(S_{j}\right)$

- $L\left(S_{j}\right)=L\left(S_{i}\left[\bar{T}_{1} / \bar{\Delta}_{1}, \ldots \bar{\Gamma}_{n} / \bar{\Delta}_{n}\right]^{l}\right)$ for $\bar{\Gamma}_{1}, \ldots \bar{\Gamma}_{n} \in L\left(S_{i}\right)$ and $\bar{\Delta}_{1}, \ldots \bar{\Delta}_{n} \in$ $L\left(S_{j}\right)$ 
- If $n>1, G \mid S_{j}$ is a (n-1)-pivot for $n$ premises $G\left|S_{j_{1}} \ldots G\right| S_{j_{n}}$, and $L\left(S_{j}\right)=L\left(S_{j_{i}}\left[\bar{\Gamma}_{1} / \bar{\Delta}_{1}, \ldots, \bar{\Gamma}_{i-1} / \bar{\Delta}_{i-1}, \bar{\Gamma}_{i+1} / \bar{\Delta}_{i+1}, \ldots, \bar{\Gamma}_{n} / \bar{\Delta}_{n}\right]^{l}\right)$ for $\bar{\Gamma}_{1}, \ldots, \bar{\Gamma}_{i-1}, \bar{\Gamma}_{i+1}, \ldots, \bar{\Gamma}_{n} \in L\left(S_{j_{i}}\right), \bar{\Delta}_{1}, \ldots, \bar{\Delta}_{i-1}, \bar{\Delta}_{i+1}, \ldots, \bar{\Delta}_{n} \in$ $L\left(S_{j}\right)$ and $i=1, \ldots, n$.

Definition 3. A completed hypersequent rule $(r)$ is convergent if for each premise $G \mid S_{i}$ one of the following conditions holds: (1) $R\left(S_{i}\right)=\emptyset$, (2) $G \mid S_{i}$ is a 0-pivot, or (3) there is a premise $G \mid S_{j}$ which is an n-pivot for $G \mid S_{i}$, with $n>0$.

Intuitively, the conclusion of a convergent rule results from a "minimal interplay" among its premises. Indeed for a premise $G \mid S_{i}$, in which $R\left(S_{i}\right)$ is not empty, two cases can arise: either the metavariables contained in it are already present in a component of the rule's conclusion, or there is a premise $G \mid S_{j}$ having this property and which allows us to obtain $G \mid S_{i}$ by suitable replacements of the metavariables.

Example 2. Convergent rules are all internal structural rules, (wnm), (lq) and $(w n m)^{n}$, see Table 3. For instance, in the particular case of $(w n m)^{2}$ (cf. Ex. 1):

- All different metavariables in the premise $P_{1}=G \mid \bar{\Gamma}_{1}^{2}, \bar{\Gamma}_{1}^{2}, \bar{\Sigma} \Rightarrow \bar{\Pi}$ are contained in the component $\bar{\Gamma}_{1}, \bar{\Sigma} \Rightarrow \bar{\Pi}$ of the conclusion. Therefore, $P_{1}$ is a 0-pivot.

- The premise $P_{1}$ is a 1-pivot for all premises $G \mid \bar{\Gamma}_{1}^{2}, \bar{\Gamma}_{i}^{2}, \bar{\Sigma} \Rightarrow \bar{\Pi}, 2 \leq i \leq 5$ as they differ from $P_{1}$ only by one metavariable.

- $P_{1}$ is a 2-pivot for the remaining premises of $(w n m)^{2}$.

Completed rules that are not convergent are (em) and (bc2).

AxiomCalc. The procedure in [4] to transform axioms into equivalent analytic (hyper)sequent rules is implemented in the PROLOG-system AxiomCalc. It takes as input any axiom provided by the user, indicates the class $\mathcal{N}_{n}$ or $\mathcal{P}_{n}$ to which the axiom belongs and, for axioms within $\mathcal{N}_{2}, \mathcal{P}_{2}$ and $\mathcal{P}_{3}$, it generates (a paper that contains) the equivalent (hyper)sequent rules. Finally, the system checks whether the generated rules are convergent.

\section{Sufficient Conditions for Density Elimination}

In this section we prove that $H M T L$ extended with any set of convergent rules admits density-elimination. Our proof uses and refines the method in [6] of density elimination by substitutions, which is outlined below: Let $d$ be a subderivation ending in the following uppermost application of density

$$
\begin{gathered}
\vdots d^{\prime} \\
\frac{G^{\prime}|\Sigma, p \Rightarrow \Pi| \Lambda \Rightarrow p}{G^{\prime} \mid \Sigma, \Lambda \Rightarrow \Pi} \text { (D) }
\end{gathered}
$$

$(D)$ is removed by substituting the occurrences of $p$ in $d$ in an "asymmetric" way, according to whether $p$ occurs in the left or in the right hand side of a 
sequent. More precisely, each component $S$ of a hypersequent in $d$ is replaced by $S\left[{ }^{p} / \Lambda\right]^{l}\left[{ }^{p} / \Sigma \Rightarrow \Pi\right]^{r}$. This way, the application of $(D)$ above is simply replaced by $(E C)$.

A problem: the resulting labeled tree, denoted by $d^{*}$, is in general not a correct derivation anymore. The reason being the presence in $d$ of external structural rules different from $(E W)$ and $(E C)$, that, by mixing the content of various conclusion components, might lead to p-axioms in their premises, i.e. to hypersequents of the form $G \mid \Theta, p^{k} \Rightarrow p$ derivable from axioms simply using weakenings; the problem is that the asymmetric substitution on a p-axiom leads, e.g., to $G \mid \Theta, \Lambda^{k}, \Sigma \Rightarrow \Pi$, which is no longer derivable in the same way.

The proof in [6]: Density-elimination was proved for calculi containing only $(E \bar{C}),(E W)$ and $(\mathrm{com})$ as external structural rules. The only problematic case was when in $d$ one of the premises of (com) led to a p-axiom. This case was handled by removing in $d^{*}$ this application of (com) and replacing it with a suitable (sub)derivation starting from the other premise.

The addition of convergent rules: We show below that, though a convergent rule $(r)$ can manipulate more hypersequent components at once and hence might create p-axioms, $(r)$ behaves well with respect to the asymmetric substitutions. Indeed, intuitively, if one or more premises of $(r)$ led in $d$ to a p-axiom, say $G \mid \Theta, p^{k} \Rightarrow p$, the special premises of $(r)$ called pivot are used to derive their substituted version $G \mid \Theta, \Lambda^{k}, \Sigma \Rightarrow \Pi$ which allow us to correctly apply $(r)$.

The length $|d|$ of a derivation $d$ is, as usual, the (maximal number of inference rules) +1 , occurring on any branch. A $(D)$-free derivation is a derivation not containing the $(D)$ rule. The following lemma, which allows us to suitably "move" multisets of formulas between components, is the key for our main proof.

Lemma 1. Given HMTL $+R$, with $R$ any set of convergent rules.

1. Any derivation $d$ of $H$ can be transformed into a derivation of $\left.H^{p} / \alpha\right]^{l}\left[{ }^{p} / \Rightarrow \alpha\right]^{r}$, for any formula $\alpha$ and propositional variable $p$.

2. Let $d^{\prime}$ and $d_{1}$ be derivations of $G^{\prime}|\Sigma, p \Rightarrow \Pi| \Lambda \Rightarrow p\left(p \notin G^{\prime}, \Sigma, \Pi, \Lambda\right)$ and $G^{\prime} \mid \Theta, \Delta \Rightarrow \Psi$. We can find a derivation of $G^{\prime}|\Theta, \Lambda \Rightarrow \Psi| \Sigma, \Delta \Rightarrow \Pi$.

Proof. 1. Just replace $p$ in $d$ everywhere with $\alpha$. The claim is proved by induction on the length of the resulting derivation, as convergent rules are completed (and hence substitutive, cf. the definition and the analogous lemma in [6]).

2. By 1 . and $d^{\prime}$ we have a derivation $d_{2}$ of $G^{\prime}|\Sigma, \odot \Delta \Rightarrow \Pi| \Lambda \Rightarrow \odot \Delta$ where $\odot \Delta$ stands for the multiplicative conjunction $\cdot$ of the formulas in $\Delta$ (note that $\left.p \notin G^{\prime}, \Sigma, \Pi, \Lambda\right)$. The desired derivation follows by applying $(C u t)$ between $G^{\prime}|\Theta, \Lambda \Rightarrow \Psi| \Delta \Rightarrow \odot \Delta$ and the end hypersequent of

$$
\begin{gathered}
\vdots d_{1} \\
\frac{G_{2}|\Sigma, \odot \Delta \Rightarrow \Pi| \Lambda \Rightarrow \odot \Delta \quad \frac{G^{\prime} \mid \Theta, \Delta \Rightarrow \Psi}{G^{\prime}|\Theta, \odot \Delta \Rightarrow \Psi| \Sigma, \odot \Delta \Rightarrow \Pi}}{\left({ }^{\prime}|\Theta, \Lambda \Rightarrow \Psi| \Sigma, \odot \Delta \Rightarrow \Pi\right.}(C u t)+(E W)
\end{gathered}
$$


We are now ready for the main theorem. Henceforth we denote by $S_{i}^{*}$ the sequent $S_{i}\left[{ }^{p} / \Lambda\right]^{l}\left[{ }^{p} / \Sigma \Rightarrow \Pi\right]^{r}$, and by $G^{*}, H^{*}$, the hypersequents $G, H$, where the same substitution is applied to each one of their components.

Theorem 1 (Density Elimination). HMTL extended with any set $R$ of convergent rules admits density elimination

Proof. To perform density elimination, it is sufficient to remove topmost applications of $(D)$. Take the derivation $d$ above in $H M T L+(D)+R$, ending in an application of $(D)$ and let $d^{\prime}$ be the (D)-free derivation ending in

$$
G^{\prime}|\Sigma, p \Rightarrow \Pi| \Lambda \Rightarrow p
$$

As convergent rules are particular completed rules, they preserve cut-elimination when added to $H M T L$. Hence we assume that $d^{\prime}$ is cut-free.

Claim: For each hypersequent $H$ in $d^{\prime}$ that is not a p-axiom, one can find a (D)-free derivation of $G^{\prime} \mid H^{*}$.

The result on density elimination follows from this claim. Just let $H$ be $G^{\prime}|\Lambda \Rightarrow p| \Sigma, p \Rightarrow \Pi$. We get that $G^{\prime}\left|G^{\prime}\right| \Lambda, \Sigma \Rightarrow \Pi \mid \Lambda, \Sigma \Rightarrow \Pi$ is derivable (note that $\left(G^{\prime}\right)^{*}=G^{\prime}$ by the eigenvariable condition on $p$ ). The desired proof of $G^{\prime} \mid \Lambda, \Sigma \Rightarrow \Pi$ follows by multiple applications of $(E C)$.

The proof of the claim proceeds by induction on the length of the cut-free subderivation $d_{H}$ of $H$ in $H M T L+R$. We distinguish cases according to the last rule $(r)$ applied in $d_{H}$. The cases $\left|d_{H}\right|=0$, or when $(r)$ is $(E C)$ or $(E W)$ are easy. The proof for logical rules and for $(\mathrm{com})$ proceeds as in [6].

Convergent rules: Assume that $(r)$ is a convergent rule of the form

$$
\frac{G\left|S_{1} \quad \ldots \quad G\right| S_{m}}{G\left|C_{1}\right| \ldots \mid C_{q}}(r)
$$

and that the conclusion of $(r)$ contains no p-axiom. We show how to find a derivation of

$$
G^{\prime}\left|G^{*}\right| C_{1}^{*}|\ldots| C_{q}^{*}
$$

Take a premise $G \mid S_{i}$. If $G \mid S_{i}$ is not a p-axiom, the inductive hypothesis gives us a derivation of $G^{\prime}\left|G^{*}\right| S_{i}^{*}$. Note that this is always the case when $R\left(S_{i}\right)=\emptyset$, and when $G \mid S_{i}$ is a 0 -pivot as in the latter case the metavariables instantiated to obtain $S_{i}$ are all included in one component of the conclusion. Thus, if $G \mid S_{i}$ was a p-axiom, the conclusion would be a p-axiom as well, thus contradicting the assumption.

Assume now that $G \mid S_{i}$ is a p-axiom. We show below that we can always obtain a $(D)$-free derivation of

$$
G^{\prime}\left|G^{*}\right| S_{i}^{*} \mid C_{s}^{*}
$$

for some $s \in\{1, \ldots, q\}$. Being $(r)$ convergent, there is an $n$-pivot premise $G \mid S_{j}$ for $G \mid S_{i}$. We show how to use $G \mid S_{j}$ to obtain the required derivation. 
- Case $n=1$ : There is a 1-pivot premise $G \mid S_{j}$ for $G \mid S_{i}$, i.e., (the metasequent leading to) $S_{i}$ differs only in 1 metavariable from (that of) $S_{j}$. By Definition $2, G \mid S_{j}$ is also a 0 -pivot and hence it is not a p-axiom. Let $G \mid S_{i}$ and $G \mid S_{j}$ be obtained as instantiations of the following premises ${ }^{4}$ of $(r)$ :

$$
S_{i} \quad \text { is obtained from } \bar{\Theta}, \bar{\Gamma}^{k}, \bar{\Delta}^{l} \Rightarrow \bar{\Pi} \text { and } S_{j} \text { from } \bar{\Theta}, \bar{\Delta}^{k+l} \Rightarrow \bar{\Pi}
$$

As $G \mid S_{i}$ is a p-axiom and $G \mid S_{j}$ is not, only $\bar{\Gamma}$ can be instantiated with a propositional variable $p$. The most general case is when $\bar{\Gamma}$ is instantiated by $\Gamma, p^{n}$, the metavariable $\bar{\Delta}$ by the multiset $\Delta, \bar{\Theta}$ by $\Theta$ and $\bar{\Pi}$ by $p$. Hence

$$
G \mid S_{i} \quad \text { is } \quad G \mid \Theta, \Gamma^{k}, \Delta^{l}, p^{m k} \Rightarrow p \quad \text { and } \quad G \mid S_{j} \quad \text { is } \quad G \mid \Theta, \Delta^{k+l} \Rightarrow p .
$$

As $G \mid S_{j}$ is not a p-axiom, by the inductive hypothesis we have a derivation for $G^{\prime}\left|G^{*}\right| \Theta, \Delta^{k+l}, \Sigma \Rightarrow \Pi$. Using the derivation $d^{\prime}$ of $G^{\prime}|\Sigma, p \Rightarrow \Pi| \Lambda \Rightarrow p$, by $k$ applications of Lemma 1.2 with $(E W)$ and $(E C)$ we get

$$
G^{\prime}\left|G^{*}\right| \Theta, \Lambda^{k}, \Delta^{l}, \Sigma \Rightarrow \Pi \mid \Sigma, \Delta \Rightarrow \Pi
$$

Now, by multiple applications of internal weakenings $(w l)$, we have

$$
G^{\prime}\left|G^{*}\right| \Theta, \Gamma^{k}, \Lambda^{m k}, \Delta^{l}, \Sigma \Rightarrow \Pi \mid \Sigma, \Delta \Rightarrow \Pi
$$

From further repeated applications of $(w l)$ on the fourth component, we finally obtain $G^{\prime}\left|G^{*}\right| S_{i}^{*} \mid C_{s}^{*}$, where $C_{s}$ stands for the component of the conclusion to which all the metavariables in (the metasequent leading to) $S_{j}$ belong.

- Assume that there is an n-pivot premise $G \mid S_{j}$ for $G \mid S_{i}$, with $n>1$. By Def. 2 (the metasequent leading to) $G \mid S_{i}$ differs from (that of) $G \mid S_{j}$ by $n$ metavariables and there exist $n$ other premises for which $G \mid S_{j}$ is an $(n-1)$ pivot. As in the previous case, let $S_{i}$ and $S_{j}$ be obtained respectively as instantiations of the following premises of $(r)$

$$
\bar{\Theta}, \bar{\Gamma}_{1}^{k_{1}}, \ldots, \bar{\Gamma}_{n}^{k_{n}}, \bar{\Delta}_{1}^{l_{1}}, \ldots, \bar{\Delta}_{n}^{l_{n}} \Rightarrow \bar{\Psi} \quad \text { and } \quad \bar{\Theta}, \bar{\Delta}_{1}^{k_{1}+l_{1}}, \ldots, \bar{\Delta}_{n}^{k_{n}+l_{n}} \Rightarrow \bar{\Psi}
$$

Assume w.l.o.g. that $G \mid S_{j}$ is:

$$
G \mid \Theta, \Delta_{1}^{k_{1}+l_{1}}, \ldots, \Delta_{n}^{k_{n}+l_{n}} \Rightarrow p
$$

Two cases have to be considered, according to the possible instantiations of the metavariables $\overline{\Gamma_{i}}$ with the propositional variable $p$ in $G \mid S_{i}$ :

(i) In $G \mid S_{i}$ all the metavariables $\overline{\Gamma_{i}}$ are instantiated with a multiset $\Gamma_{i}$ together with at least one occurrence of $p$. Then we repeatedly apply Lemma 1.2 to $d^{\prime}$ and $G \mid S_{j}$ together with $(E W)$ and $(E C)$ to replace $\Delta_{1}, \ldots, \Delta_{n}$ with $\Lambda$ in $S_{j}$, respectively $k_{1}, \ldots k_{n}$ times. This way we get $G\left|\Theta, \Lambda^{k_{1}}, \ldots, \Lambda^{k_{n}}, \Delta_{1}^{l_{1}}, \ldots, \Delta_{n}^{l_{n}}, \Sigma \Rightarrow \Pi\right| \Delta_{1}, \Sigma \Rightarrow \Pi|\ldots| \Delta_{n}, \Sigma \Rightarrow \Pi$. The desired hypersequent $G^{\prime}\left|G^{*}\right| S_{i}^{*} \mid C_{s}^{*}$ follows by suitable applications of $(w l)$ and (EC) (as in the 1-pivot case, $C_{s}$ stands for the component of the conclusion to which all the metavariables in $S_{j}$ belong).

\footnotetext{
${ }^{4}$ To simplify the notation $\bar{\Theta}$ stands for all the metavariables in common, except $\bar{\Delta}$.
} 
(ii) In $G \mid S_{i}$ all the metavariables $\overline{\Gamma_{i}}$ are instantiated with $\Gamma_{i}, p^{m_{i}}$ and $m_{i}>0$ only for $r$ of them $(1 \leq r<n)$. (Note that in this case Lemma 1.2 would replace each metavariable $\Delta_{i}$ with $\Lambda$, leading to at least $n$ occurrences of $\Lambda$, and $n>r)$. The idea here is to find another premise of $(r)$ which is not a p-axiom, for which suitable applications of Lemma 1.2 do the job. The existence of (at least one) such a premise is guaranteed by the notion of $n$-pivot.

We first illustrate the way we proceed with an example for $n=3$.

Assume that $S_{i}$ arises as an instantiation of $\bar{\Gamma}_{1}, \bar{\Gamma}_{2}, \bar{\Gamma}_{3} \Rightarrow \bar{\Pi}$ and $S_{j}$ as an instantiation of $\bar{\Delta}_{1}, \bar{\Delta}_{2}, \bar{\Delta}_{3} \Rightarrow \bar{\Pi}\left(G \mid S_{j}\right.$ is a 3 -pivot for $\left.G \mid S_{i}\right)$. By definition of 3-pivot, there exist 3 premises in $(r)$ for which $G \mid S_{j}$ is a 2pivot. For each of these premises, there exist 2 premises in $(r)$ for which $G \mid S_{j}$ is a 1-pivot. In the figure below we show how all these premises are related w.r.t the metavariables they instantiate.

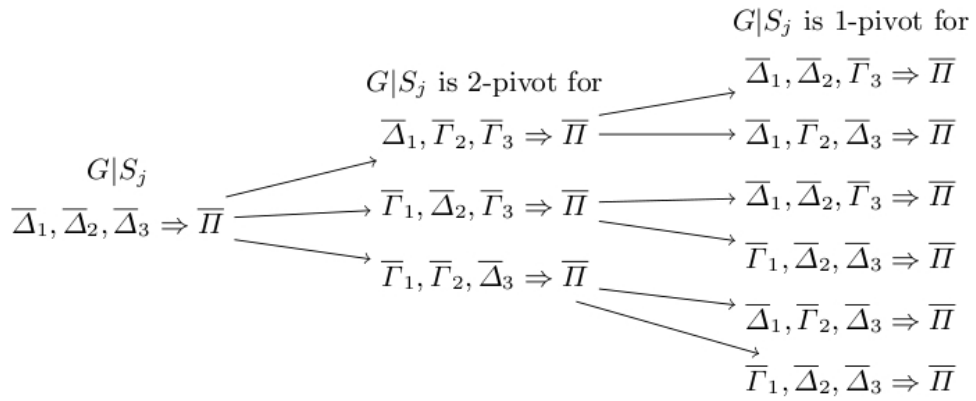

(Case $r=1$ ) If only 1 metavariable, say $\bar{\Gamma}_{1}$, is instantiated in $S_{i}$ with $\Gamma_{1}, p$ we need to find a corresponding premise which will not contain a p-axiom, i.e., that does not contain $\bar{\Gamma}_{1}$. The first occurrence of such a premise is among the premises that have $G \mid S_{j}$ as a 2-pivot, that is $\bar{\Delta}_{1}, \bar{\Gamma}_{2}, \bar{\Gamma}_{3} \Rightarrow \bar{\Pi}$.

(Case $r=2$ ) Assume now that 2 metavariables, say $\bar{\Gamma}_{1}, \bar{\Gamma}_{2}$, are instantiated with $\Gamma_{1}, p$ and $\Gamma_{2}, p$, respectively. Again, we need to find a corresponding premise that is not a p-axiom. In this case, the set of premises that have $G \mid S_{j}$ as a 2-pivot does not suffice because each of them contains either $\bar{\Gamma}_{1}$ or $\bar{\Gamma}_{2}$. The first occurrence of a premise that is not a p-axiom is among the premises that have $G \mid S_{j}$ as a 1-pivot, i.e., $\bar{\Delta}_{1}, \bar{\Delta}_{2}, \bar{\Gamma}_{3} \Rightarrow \bar{\Pi}$.

In general, we can eventually find a premise that is not a p-axiom among those that have $G \mid S_{j}$ as $(n-r)$-pivot. Assume for the general case that the occurrences of $p$ in $G \mid S_{i}$ are related to the instantiation of $r$ different metavariables, w.l.o.g $\overline{\Gamma_{1}}, \ldots, \overline{\Gamma_{r}}$, i.e., $S_{i}$ is

$\Theta, \Gamma_{1}^{k_{1}}, \ldots, \Gamma_{n}^{k_{n}}, \Delta_{1}^{l_{1}}, \ldots, \Delta_{n}^{l_{n}}, p^{m_{1} k_{1}+\ldots+m_{r} k_{r}} \Rightarrow p \quad$ with $m_{1}, \ldots, m_{r}>0$

Then we can find premises for which $G \mid S_{j}$ is an $(n-r)$-pivot; (the metasequent leading to) those premises differ from that of $G \mid S_{i}$ in $r$ metavariables. (At least) one of these premises will not be a p-axiom, and hence 
it will have the form:

$$
G \mid \Theta, \Delta_{1}^{k_{1}+l_{1}}, \ldots, \Delta_{r}^{k_{r}+l_{r}}, \Gamma_{r+1}^{k_{r+1}}, \Delta_{r+1}^{l_{r+1}}, \ldots, \Gamma_{n}^{k_{n}}, \Delta_{n}^{l_{n}} \Rightarrow p
$$

By inductive hypothesis we have a derivation of:

$$
G^{\prime}\left|G^{*}\right| \Theta, \Delta_{1}^{k_{1}+l_{1}}, \ldots, \Delta_{r}^{k_{r}+l_{r}}, \Gamma_{r+1}^{k_{r+1}}, \Delta_{r+1}^{l_{r+1}}, \ldots, \Gamma_{n}^{k_{n}}, \Delta_{n}^{l_{n}}, \Sigma \Rightarrow \Pi
$$

Then we repeatedly apply Lemma 1.2 together with $(E W)$ and $(E C)$ to replace $\Delta_{1}, \ldots, \Delta_{r}$ with $\Lambda$, respectively $k_{1}, \ldots, k_{r}$ times. After suitable applications of internal weakening and further external contractions, we finally get

$$
G^{\prime}\left|G^{*}\right| S_{i}^{*} \mid C_{s}^{*}
$$

In summary, when the last rule in $d_{H}$ is convergent, for each premise $G \mid S_{i}$ we have:

- If $G \mid S_{i}$ does not contain any p-axiom, $G^{\prime}\left|G^{*}\right| S_{i}^{*}$ is (D)-free derivable.

- If $G \mid S_{i}$ contains a p-axiom, then $G^{\prime}\left|G^{*}\right| S_{i}^{*} \mid C_{s}^{*}$ is (D)-free derivable.

The required derivation of $G^{\prime}\left|G^{*}\right| C_{1}{ }^{*}|\ldots| C_{q}{ }^{*}$ follows by $(r)$ and subsequent applications of $(E C)$, if needed. This completes the proof of the main claim.

\section{From Density Elimination to Standard Completeness}

Theorem 1 together with the results in $[3,13]$ lead to standard completeness for any logic $\mathbf{L}$ extending MTL with any set $\mathcal{A}$ of axioms having equivalent convergent rules. (For all concepts of universal algebra below we refer to $[3,9,13]$ ).

As shown in [13], density elimination is indeed a uniform method to establish rational completeness for any extension of MTL. From Theorem 1 we can therefore state the following: let $\mathcal{L}$ be an $M T L$-algebra (see [8] and steps 1-4 in the introduction) satisfying the equations $\mathcal{A}^{*}$ corresponding to the axioms in $\mathcal{A}$

A formula $\alpha$ is satisfied in each dense $\mathcal{L}$-chain $\Leftrightarrow \alpha$ is derivable in $\mathbf{M T L}+\mathcal{A}$.

Standard completeness is then achieved through so called Dedekind Mac-Neille completion, which generalizes to various ordered algebraic structures Dedekind's embedding of the rational numbers into the reals.

It is shown, e.g. in [13], that the Dedekind-McNeille completion of a dense $M T L$-chain is still a dense $M T L$-chain (in other words, it is preserved by Dedekind-MacNeille-completion). It is easy to see that the results in [3] on the preservation of equations by this completion hold for the equations $\mathcal{A}^{*}$ when restricting to $M T L$-chains. Hence the Dedekind-MacNeille completion of a dense $\mathcal{L}$-chain is still a dense $\mathcal{L}$-chain, and in addition it is order-isomorphic to $[0,1]$. This leads to standard completeness for the logic $\mathbf{L}$. 
Remark Our sufficient condition for density-elimination can be easily extended to first-order calculi, hence leading, by the results in [6], to standard completeness proofs for axiomatic extensions of first-order MTL. In contrast, convergency of rules might be too weak to ensure density-elimination for hypersequent calculi not containing $(w l)$ and $(w r)$. For these calculi, formalizing logics which extend Uninorm Logic UL [13], only calculi-tailored proofs of density-elimination are available; the proof in [6] applies indeed to very few of them, namely, those extending the calculus for UL only with additional internal structural rules having a very simple structure (e.g. contraction $(c)$ is not one of them).

\subsection{A case study}

As a corollary of our results follows that the family of logics obtained by extending MTL with the axioms $(w n m)^{n}: \neg(\alpha \cdot \beta)^{n} \vee\left((\alpha \wedge \beta)^{n-1} \rightarrow(\alpha \cdot \beta)^{n}\right)$, for $n \geq 2$ (here $x^{n}$ stands for $x \cdots \cdots x, n$ times) are standard complete and hence they are fuzzy logics in the sense of [11]. This new family of logics, discovered by playing with AxiomCalc, contains infinitely many different logics. This can be easily seen by noticing that $(w n m)^{n}$ is valid in the $m$-valued logic of Eukasiewicz if and only if $m \leq n+1$.

Acknowledgments Work supported by the FWF START Y544-N23 and the WWTF project MA07-016.

\section{References}

1. A. Avron. The method of hypersequents in the proof theory of propositional nonclassical logics. In W. Hodges et al. editors, Logic: from foundations to applications. Proc. Logic Colloquium, Keele, UK, 1993, pages 1-32, 1996.

2. M. Baaz and R. Zach. Hypersequents and the proof theory of intuitionistic fuzzy logic. Proc. of Computer Science Logic (CSL'2000). LNCS 1862, Springer, 187-201. 2000.

3. A. Ciabattoni, N. Galatos and K. Terui. MacNeille Completions of FL-algebras. Algebra Universalis, 66(4): 405-420, 2011.

4. A. Ciabattoni, N. Galatos and K. Terui. From axioms to analytic rules in nonclassical logics. Proceedings of LICS 2008, pp. 229-240, 2008.

5. A. Ciabattoni and F. Esteva and L. Godo. T-norm based logics with $n$-contraction. Neural Network World, 12(5): 441-453, 2002.

6. A. Ciabattoni and G. Metcalfe. Density elimination. Theor. Comput. Sci., 403(2-3): 328-346, 2008.

7. F. Esteva, J. Gispert, L. Godo and F. Montagna. On the Standard and Rational Completeness of some Axiomatic Extensions of the Monoidal T-norm Logic, Studia Logica, 71(2): 199-226, 2002.

8. F. Esteva and L. Godo. Monoidal t-norm based logic: towards a logic for leftcontinuous t-norms. Fuzzy Sets and Systems, 124:271-288, 2001.

9. N. Galatos, P. Jipsen, T. Kowalski and H. Ono. Residuated Lattices: an algebraic glimpse at substructural logics, Studies in Logics and the Foundations of Mathematics, Elsevier, 2007. 
10. S. Jenei and F. Montagna. A proof of standard completeness for Esteva and Godo's MTL logic, Studia Logica, 70(2): 183-192, 2002.

11. P. Hájek. "Metamathematics of Fuzzy Logic", Kluwer. 1998.

12. R. Horcik. Alternative Proof of Standard Completeness Theorem for MTL, Soft Computing, 11(2), 2006.

13. G. Metcalfe and F. Montagna. Substructural fuzzy logics. Journal of Symbolic Logic, 7(3):834-864, 2007.

14. G. Metcalfe and N. Olivetti and D. Gabbay. "Proof Theory for Fuzzy Logics", Springer Series in Applied Logic Vol. 36, 2008.

15. F. Montagna and H. Ono. Kripke semantics, undecidability and standard completeness for Esteva and Godo's logic MTL $\forall$. Studia Logica, 71(2):227-245, 2002.

16. H. Ono and K. Komori. Logics without the contraction rule. Journal of Symbolic Logic, 50: 169-201, 1985.

17. G. Takeuti and T. Titani. Intuitionistic fuzzy logic and intuitionistic fuzzy set theory. Journal of Symbolic Logic, 49(3):851-866, 1984.

\section{A Appendix}

The normal form of axioms within the classes $\mathcal{N}_{2}, \mathcal{P}_{2}$ and $\mathcal{P}_{3}$ is the following.

$\mathcal{N}_{2}$ : Axioms have the form $\bigwedge_{1 \leq i \leq n} \delta_{i}$, in which every $\delta_{i}$ is a $\alpha_{1} \cdots \alpha_{m} \rightarrow \beta$ where:

$-\beta=0$ or $\beta_{1} \vee \cdots \vee \beta_{k}$ and each $\beta_{l}$ is a multiplicative conjunction of propositional variables and

- each $\alpha_{i}$ is of the form $\bigwedge_{1 \leq j \leq p} \gamma_{i}^{j} \rightarrow \beta_{i}^{j}$ where

$\circ \beta_{i}^{j}=0$ or a propositional variable, and

$\circ \gamma_{i}^{j}$ is a multiplicative conjunction or a disjunction of propositional variables (or 1).

$\mathcal{P}_{2}$ : Axioms have the form $\bigvee_{1 \leq i \leq n} \delta_{i}$, where each $\delta_{i}$ is of the form

$\bigwedge_{1 \leq j \leq m} \alpha_{j} \rightarrow \beta_{j}$ or $\bigwedge_{1 \leq j \leq m} \alpha_{j}$ where:

- each $\alpha_{j}$ is a multiplicative conjunction or disjunction of propositional

variables and 1 , and

$-\beta_{j}=0$ or a propositional variable.

$\mathcal{P}_{3}$ : Axioms have the form $\delta_{1} \vee \cdots \vee \delta_{n}$, where each $\delta_{i}$ is in $\mathcal{N}_{2}$. 